\title{
A Solvable Tensor Field Theory
}

\author{
R. Pascalie* \\ Université de Bordeaux, LaBRI, CNRS UMR 5800, Talence, France, EU \\ Mathematisches Institut der Westfälischen Wilhelms-Universität, Münster, Germany, EU
}

August 3, 2020

\begin{abstract}
We solve the closed Schwinger-Dyson equation for the 2-point function of a tensor field theory with a quartic melonic interaction, in terms of Lambert's W-function, using a perturbative expansion and Lagrange-Bürmann resummation. Higher-point functions are then obtained recursively.
\end{abstract}

\section{Introduction}

Tensor models have regained a considerable interest since the discovery of their large $N$ limit (see [1], [2], [3] or the book [4]). Recently, tensor models have been related in [5] and [6], to the Sachdev-Ye-Kitaev model [7], [8], [9], [10], which is a promising toy-model for understanding black holes through holography (see also [11], [12], the lectures [13] and the review [14]).

In this paper we study a specific type of tensor field theory (TFT) ${ }^{1}$. More precisely, we consider a $U(N)$-invariant tensor models whose kinetic part is modified to include a Laplacianlike operator (this operator is a discrete Laplacian in the Fourier transformed space of the tensor index space). This type of tensor model has originally been used to implement renormalization techniques for tensor models (see [16], the review [17] or the thesis [18] and references within) and has also been studied as an SYK-like TFT [19]. Recently, the functional Renormalization Group (FRG) as been used in 20] to investigate the existence of a universal continuum limit in tensor models, see also the review 21]. This is also closely related to the Polchinski's equation for TFT [22]. Our study provides a complementary non-perturbative tool to these two approaches.

The Ward-Takahashi identity (WTI) for TFT, first appeared in [23] and has been fully established in [24]. It was used in [25] to derive the tower of exact Schwinger-Dyson equations (SDE) with connected boundary graph. Their large $N$ limit was established in [25]. Then the tower of SDE with a disconnected boundary graph was derived in [26]. Numerical methods were used in 27] for a $\phi_{5}^{4}$ just renormalizable tensor model to study the solutions of closed SDE for the 2- and 4-point functions.

Let us also mention here that the WTI has been already successfully used to study the SDE in the context of matrix models of non-commutative quantum field theory - see [28] and [29].

*romain.pascalie@u-bordeaux.fr

${ }^{1}$ Not to be confused with tensor fields living on a space-time such as in 15 
In particular, the closed SDE 2-point function for the non-commutative and 2 dimensional $\lambda \phi^{4}$ has been solved in [30] using and resumming a perturbative expansion. The building block of this solution is the Lambert- $W$ function.

Our paper is organised as follows. In the following section we describe the setup of our work, namely the action of the model, the boundary graph expansion of the free energy and the 2-point function SDE in the large $N$ limit. The third section is dedicated to the analysis of the perturbative expansion of the 2-point function which leads us to consider the model with one quartic melonic interaction. In the fourth section we perform the resummation of the perturbative expansion, in order to obtain the non-perturbative solution of the SDE. We then discuss shortly the higher-point functions before giving some concluding remarks. In the appendix we obtain recurrence relations on the number appearing in the perturbative expansion which translate into formulas involving Stirling numbers.

\section{The model}

Let us consider a complex rank-3 bosonic tensor field theory with an action of the form

$$
\mathcal{S}[\varphi, \bar{\varphi}]=\sum_{\mathbf{x}} \bar{\varphi}^{\mathbf{x}}\left(1+|\mathbf{x}|^{2}\right) \varphi^{\mathbf{x}}+\frac{\lambda}{N^{2}} \sum_{c=1}^{3} \sum_{\mathbf{a}, \mathbf{b}} \bar{\varphi}^{\mathbf{a}} \varphi^{\mathbf{b}_{\hat{c}} a_{c}} \bar{\varphi}^{\mathbf{a}_{\hat{c}} b_{c}} \varphi^{\mathbf{a}}
$$

with $\mathbf{x}=\left(x_{1}, x_{2}, x_{3}\right) \in\left\{\frac{1}{N}, \frac{2}{N}, \ldots, 1\right\}^{3},|\mathbf{x}|^{2}=x_{1}^{2}+x_{2}^{2}+x_{3}^{2}$ and $\mathbf{a}_{\hat{c}} b_{c}=\left(a_{1}, \ldots, a_{c-1}, b_{c}, a_{c+1}, \ldots, a_{D}\right)$ for a $D$-tuple. Here $\varphi$ is a rank-3 bosonic tensor. Note that the quartic melonic interaction terms in the action, also called pillows, are invariant under $\mathrm{U}(N)^{3}$. The tensor fields transform as

$$
\varphi^{\mathbf{x}} \rightarrow \varphi^{\mathbf{x}}=\sum_{y_{c}} U_{x_{c} y_{c}}^{(c)} \varphi^{\mathbf{x}_{\hat{c}} y_{c}}, \quad \bar{\varphi}^{\mathbf{x}} \rightarrow \bar{\varphi}^{\mathbf{x}}=\sum_{y_{c}} \bar{U}_{x_{c} y_{c}}^{(c)} \bar{\varphi}^{\mathbf{x}_{c} y_{c}}
$$

for $U^{(c)} \in \mathrm{U}(N)$ and for each colour $c \in\{1,2,3\}$. Each copy of the group $\mathrm{U}(N)$ acts on only one index of the tensor. Thus, the indices of the tensors have no symmetries and only indices of the same colour can be contracted.

Let us emphasise that the kinetic term above represents the discrete Laplacian in the Fourier transformed of the tensor index space and a mass term which regularises the IR divergences.

The generating functional of the model is

$$
\mathrm{Z}[J, \bar{J}]=\int \mathcal{D} \varphi \mathcal{D} \bar{\varphi} \exp \left(-\mathcal{S}[\varphi, \bar{\varphi}]+\sum_{\mathbf{x}}\left(\bar{J}_{\mathbf{x}} \varphi^{\mathbf{x}}+J_{\mathbf{x}} \bar{\varphi}^{\mathbf{x}}\right)\right) .
$$

In tensor models, Feynman graphs (see Fig. 1) can be drawn with two types of lines: dotted lines representing the propagator and solid lines which correspond to the contractions of the index of the tensors in the interaction. Hence, each solid line has a colour which correspond to the contracted index of the tensor. A colouring of a graph is then an edge-colouring where the solid lines have colours in $\{1,2,3\}$ and the dotted lines have the colour 0 . The Feynman graphs are then 4-coloured graphs. We consider a complex tensor field theory so the graphs are bipartite.

Moreover each Feynman graph has a boundary graph which is defined as follows: to each external leg of a Feynman graph is associated an external vertex so that the open graph is bipartite. These vertices are exactly the vertices of the boundary graph. An edge of colour $c$ in the boundary graph, corresponds to a path between two external legs in the Feynman 
a)

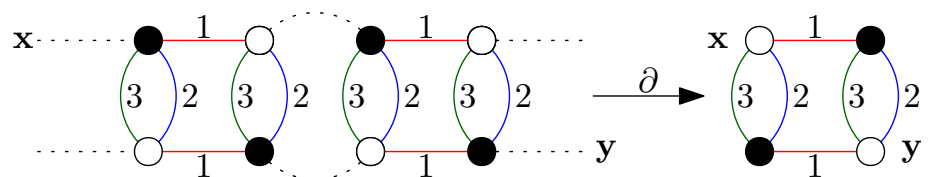

b)

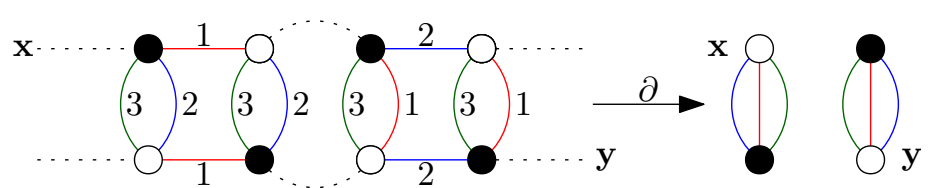

Figure 1: Two connected Feynman graphs and the associated boundary graphs in the tensor field theory (1). White and black vertex in a boundary graph $\mathcal{B}$, correspond in $\mathbb{J}(\mathcal{B})$ to the sources $J$ and $\bar{J}$ respectively. In the figure a) the boundary graph $V_{1}$ is connected and in fig. b) the boundary graph $\mathrm{m} \mid \mathrm{m}$ is disconnected.

graph, which alternates between dotted lines and lines of colour $c$. The boundary graphs are then 3-coloured graphs, only composed of solid lines. A more detailed exposition of boundary graphs can be found in [24] and 25].

The connected $2 k$-point functions are then split into sectors indexed by a boundary graph $\mathcal{B}$, and taken to be

$$
\mathrm{G}_{\mathcal{B}}^{(2 k)}(\mathbf{X})=\left.\frac{N^{-\alpha(\mathcal{B})}}{\mathrm{Z}_{0}} \prod_{i=1}^{k}\left(\frac{\delta}{\delta \bar{J}_{\mathbf{p}^{i}}} \frac{\delta}{\delta J_{\mathbf{x}^{i}}}\right) \mathrm{Z}[J, \bar{J}]\right|_{J=\bar{J}=0},
$$

where $Z_{0}=Z[0,0], \mathbf{X}=\left(\mathbf{x}^{1}, \ldots, \mathbf{x}^{k}\right) \in\left\{\frac{1}{N}, \frac{2}{N}, \ldots, 1\right\}^{3 k}$ so that for all $c \in\{1,2,3\}$ and $(i, j) \in\{1, \ldots, k\}^{2}, x_{c}^{i} \neq x_{c}^{j}$.

The $\mathbf{p}^{i} \in\left\{\frac{1}{N}, \frac{2}{N}, \ldots, 1\right\}^{3}$ are momentum 3-tuples depending on the coordinates $\mathbf{X}$ in a way constrained by the boundary graph $\mathcal{B}$. Hence the $2 k$-point functions do not depend on the $\mathbf{p}^{i}$ but only on $\mathbf{X}$. For instance, for the boundary graph $V_{1}$ (see Fig. 1), $\mathbb{J}\left(V_{1}\right)\left(\mathbf{x}^{1}, \mathbf{x}^{2}\right)=$ $J_{\mathbf{x}^{1}} J_{\mathbf{x}^{2}} \bar{J}_{\mathbf{p}^{1}} \bar{J}_{\mathbf{p}^{2}}=J_{\mathbf{x}^{1}} J_{\mathbf{x}^{2}} \bar{J}_{x_{1}^{1} x_{2}^{2} x_{3}^{2}} \bar{J}_{x_{1}^{2} x_{2}^{1} x_{3}^{1}}$, where $\mathbf{p}^{1}=\left(x_{1}^{1}, x_{2}^{2}, x_{3}^{2}\right)$ and $\mathbf{p}^{2}=\left(x_{1}^{2}, x_{2}^{1}, x_{3}^{1}\right)$. Let us note that white and black vertices in a boundary graph $\mathcal{B}$, correspond in $\mathbb{J}(\mathcal{B})$ to the sources $J$ and $\bar{J}$ respectively.

We also introduce the scalings $\alpha(\mathcal{B})$ for each boundary graph $\mathcal{B}$, note that they do not depend on the choice of colouring of the respective graph $\mathcal{B}$. For example, the three pillow graphs have the same scaling $\alpha\left(V_{1}\right)=\alpha\left(V_{2}\right)=\alpha\left(V_{3}\right)$.

The free energy is written as an expansion over boundary graphs (see again 24 for more details):

$$
\mathrm{W}[J, \bar{J}]=\sum_{k=1}^{\infty} \sum_{\substack{\mathcal{B} \in \partial_{\mathcal{S}_{\text {int }}} \\ V(\mathcal{B})=2 k}} \sum_{\mathbf{X}} \frac{N^{\alpha(\mathcal{B})}}{|\operatorname{Aut}(\mathcal{B})|} \mathrm{G}_{\mathcal{B}}^{(2 k)}(\mathbf{X}) \cdot \mathbb{J}(\mathcal{B})(\mathbf{X}),
$$

where $\partial_{\mathcal{S}_{\text {int }}}$ is the set of boundary graphs associated to the interaction terms, $V(\mathcal{B})$ is the number of vertices of $\mathcal{B}$ and we note $\mathbb{J}(\mathcal{B})(\mathbf{X})=J_{\mathbf{x}^{1}} \ldots J_{\mathbf{x}^{k}} \bar{J}_{\mathbf{p}^{1}} \ldots \bar{J}_{\mathbf{p}^{k}}$. Here $\operatorname{Aut}(\mathcal{B})$ is the symmetry group of the graph $\mathcal{B}$, which namely consists of all graph-automorphisms that preserve the bipartiteness in a strict sense - black vertices are mapped to black vertices, white to white and respect the colour on edges (see [24, Def. 7 and examples]).

In [25], we used the Ward-Takahashi identity established in [24] to determine the SchwingerDyson equation for $2 k$-point function with a connected boundary graph $\mathcal{B}$. Then in [31], requiring that the SDE for connected boundary graph have a well-defined large $N$ limit, we 
conjectured a general formula for the scalings

$$
\alpha(\mathcal{B})=3-B-2 g-2 k,
$$

where $2 k$ is the number of vertices of $\mathcal{B}, B$ is its number of connected components and $g$ its genus. The genus of a graph is the minimal integer $g$ such that the graph can be drawn without crossings on a surface of genus $g$. In particular, the SDE for the 2-point function is

$$
\begin{aligned}
& \mathrm{G}^{(2)}(\mathbf{x})=\frac{1}{|\mathbf{x}|^{2}}-\frac{2 \lambda}{|\mathbf{x}|^{2}} \sum_{a=1}^{3}\left(\frac{1}{N^{2}} \sum_{\mathbf{q}_{\hat{a}}} \mathrm{G}^{(2)}\left(\mathbf{q}_{\hat{a}} x_{a}\right) \mathrm{G}^{(2)}(\mathbf{x})+\frac{1}{N^{4}} \mathrm{G}_{a}^{(4)}(\mathbf{x}, \mathbf{x})\right. \\
& \left.+\frac{1}{N^{5}} \sum_{\mathbf{q}_{\hat{a}}} \mathrm{G}_{\mathrm{m} \mid \mathrm{m}}^{(4)}\left(\mathbf{q}_{\hat{a}} x_{a}, \mathbf{x}\right)+\frac{1}{N^{2}} \sum_{q_{a}} \frac{\mathrm{G}^{(2)}\left(\mathbf{x}_{\hat{a}} q_{a}\right)-\mathrm{G}^{(2)}(\mathbf{x})}{x_{a}^{2}-q_{a}^{2}}+\frac{1}{N^{4}} \sum_{c \neq a} \sum_{q_{b}} \mathrm{G}_{c}^{(4)}\left(\mathbf{x}, \mathbf{x}_{\hat{b}} q_{b}\right)\right),
\end{aligned}
$$

where in the last term $b \neq c$ and $b \neq a$. Following 31 , for $N=\frac{\tilde{N}}{\Lambda}$ and using

$$
\lim _{\tilde{N} \rightarrow \infty} \frac{\Lambda}{\tilde{N}} \sum_{k=1}^{\tilde{N}} f\left(\frac{k \Lambda}{\tilde{N}}\right)=\int_{0}^{\Lambda} \mathrm{d} x f(x)
$$

the SDE for the 2-point function writes

$$
\mathrm{G}^{(2)}(\mathbf{x})=\left(1+|\mathbf{x}|^{2}+2 \lambda \sum_{c=1}^{3} \int_{0}^{\Lambda} \mathrm{d} \mathbf{q}_{\hat{c}} \mathrm{G}^{(2)}\left(\mathbf{q}_{\hat{c}} x_{c}\right)\right)^{-1},
$$

where $\mathrm{d} \mathbf{q}_{\hat{c}}=\prod_{d \neq c} \mathrm{~d} q_{d}$. The aim of this paper is to solve the 2-point function of this type of models, in the limit $\Lambda=\infty$. We will mainly study the case with $c=1$ only, which is essential for solving the SDE, as we will see in the next section.

\section{Perturbative expansion}

In this section we will to compute the first orders of the perturbative expansion of the 2point function. We use a Taylor subtraction scheme to renormalize the UV divergences. For simplicity, let us plug in equation (9), the following expansion of the 2-point function

$$
\mathrm{G}^{(2)}(\mathbf{x})=\sum_{n \geq 0} \lambda^{n} \mathrm{G}_{n}^{(2)}(\mathbf{x})
$$

in order to obtain a recursive equation for $n \geq 1$, which writes:

$$
\mathrm{G}_{n}^{(2)}(\mathbf{x})=-\frac{2}{|\mathbf{x}|^{2}+1} \sum_{c=1}^{3} \int \mathrm{d} \mathbf{\mathbf { q } _ { \hat { c } }} \sum_{k=0}^{n-1}\left(\mathrm{G}_{k}^{(2)}\left(\mathbf{q}_{\hat{c}} x_{c}\right)-\frac{\delta_{k 0}}{1+\left|\mathbf{q}_{\hat{c}}\right|^{2}}\right) \mathrm{G}_{n-k-1}^{(2)}(\mathbf{x}),
$$

where $\left|\mathbf{q}_{\hat{c}}\right|^{2}=\sum_{d \neq c} q_{d}^{2}$, the integration on $q_{c}$ is over $[0, \infty]$, and when $k=0$ we subtract the first Taylor term to regularise the divergent integration on the free propagator $G_{0}^{(2)}$. 


\subsection{Model with the 3 quartic melonic interactions}

Using the recursive equation (11), we get

$$
\begin{aligned}
\mathrm{G}_{0}^{(2)}(\mathbf{x}) & =\frac{1}{1+|\mathbf{x}|^{2}}, \\
\mathrm{G}_{1}^{(2)}(\mathbf{x}) & =-\frac{2}{\left(1+|\mathbf{x}|^{2}\right)^{2}} \sum_{c=1}^{3} \int \mathrm{d} \mathbf{q}_{\hat{c}}\left(\frac{1}{1+\left|\mathbf{q}_{\hat{c}} x_{c}\right|^{2}}-\frac{1}{1+\left|\mathbf{q}_{\hat{c}}\right|^{2}}\right) \\
& =\frac{\pi}{2\left(1+|\mathbf{x}|^{2}\right)^{2}} \sum_{c=1}^{3} \log \left(x_{c}^{2}+1\right), \\
\mathrm{G}_{2}^{(2)}(\mathbf{x}) & =-\frac{2}{1+|\mathbf{x}|^{2}} \sum_{c=1}^{3} \int \mathrm{d} \mathbf{\mathbf { q } _ { \hat { c } }}\left\{\left(\frac{1}{1+\left|\mathbf{q}_{\hat{c}} x_{c}\right|^{2}}-\frac{1}{1+\left|\mathbf{q}_{\hat{c}}\right|^{2}}\right) \frac{\pi}{2\left(1+|\mathbf{x}|^{2}\right)^{2}} \sum_{d=1}^{3} \log \left(x_{d}^{2}+1\right)\right. \\
& \left.+\frac{1}{1+|\mathbf{x}|^{2}} \frac{\pi}{2\left(1+\left|\mathbf{q}_{\hat{c}} x_{c}\right|^{2}\right)^{2}} \sum_{d=1}^{3} \log \left(\left(\mathbf{q}_{\hat{c}} x_{c}\right)_{d}+1\right)\right\}, \\
& =\frac{1}{\left(1+|\mathbf{x}|^{2}\right)^{2}}\left(\sum_{c=1}^{3} \sum_{d=1}^{3} \frac{\pi^{2} \log \left(x_{c}^{2}+1\right) \log \left(x_{d}^{2}+1\right)}{4\left(1+|\mathbf{x}|^{2}\right)}-\sum_{c=1}^{3} \frac{\pi \log \left(x_{c}^{2}+1\right)}{2\left(x_{c}^{2}+1\right)}\right. \\
& -\pi^{2} \sum_{c=1}^{3} \frac{x_{c} \log \left(\frac{1}{4}\left(x_{c}^{2}+1\right)\right)+2 \tan ^{-1}\left(x_{c}\right)}{2\left(x_{c}^{3}+x_{c}\right)} .
\end{aligned}
$$

We can remark that the last term in $\mathrm{G}_{2}^{(2)}(\mathbf{x})$ is the only term not containing powers of logarithms. It comes from the last term of $(14)$ for $d \neq c$, which graphically corresponds to figure 2. This suggests that if we look at a model with only 1 pillow interaction, such graphs cannot

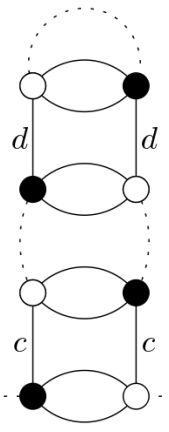

Figure 2: The only graphs at 2-loop order, giving contribution other than powers of logarithms for $d \neq c$.

exist, and the perturbative expansion should only be made of powers of logarithms.

\subsection{Model with 1 quartic melonic interaction}

Indeed, for only the pillow for the colour 1 as an interaction, we get:

$\mathrm{G}^{(2)}(\mathbf{x})=\frac{1}{1+|\mathbf{x}|^{2}}+\frac{\pi \lambda}{2\left(1+|\mathbf{x}|^{2}\right)^{2}} \log \left(x_{1}^{2}+1\right)+\frac{(\pi \lambda)^{2}}{4\left(1+|\mathbf{x}|^{2}\right)^{2}}\left(\frac{\log ^{2}\left(x_{1}^{2}+1\right)}{\left(1+|\mathbf{x}|^{2}\right)}-\frac{\log \left(x_{1}^{2}+1\right)}{\left(x_{1}^{2}+1\right)}\right)+O\left(\lambda^{3}\right)$. 
In this case, we can notice that only two types of integrals appear:

$$
\begin{gathered}
\int \mathrm{d} \mathbf{q}_{\hat{1}}\left(\frac{1}{1+\left|\mathbf{q}_{\hat{1}} x_{1}\right|^{2}}-\frac{1}{1+\left|\mathbf{q}_{\hat{1}}\right|^{2}}\right)=-\frac{\pi}{4} \log \left(x_{1}^{2}+1\right), \\
\int \mathrm{d} \mathbf{q}_{\hat{1}} \frac{1}{\left(1+\left|\mathbf{q}_{\hat{1}} x_{1}\right|^{2}\right)^{n}}=\frac{\pi\left(1+x_{1}^{2}\right)^{1-n}}{4(n-1)} \quad \text { for } n>1 .
\end{gathered}
$$

Hence, we can compute easily higher orders in the loop expansion 2 , which suggest the following form for all order $n$ in the coupling

$$
\mathrm{G}_{n}^{(2)}(\mathbf{x})=\left(\frac{\pi}{2}\right)^{n}\left(\frac{\log ^{n}\left(1+x_{1}^{2}\right)}{\left(1+|\mathbf{x}|^{2}\right)^{n+1}}+\frac{(-1)^{n}}{\left(1+x_{1}^{2}\right)^{n}} \sum_{k=1}^{n-1}(-1)^{k} \log ^{k}\left(1+x_{1}^{2}\right) \sum_{m=1}^{k} a_{n, k, m} \frac{\left(1+x_{1}^{2}\right)^{m}}{\left(1+|\mathbf{x}|^{2}\right)^{m+1}}\right)
$$

where we conjecture that the numbers $a_{n, k, m}$ are

$$
\begin{aligned}
a_{n, k, m} & =\left(\begin{array}{c}
n-1 \\
m-1
\end{array}\right) \frac{m !}{k !}\left|s_{n-m, n-k}\right| \\
& =(-1)^{k-m}(n-1) ! m \frac{s_{n-m, n-k}}{(n-m) ! k !},
\end{aligned}
$$

where $s_{n, k}$ are the Stirling numbers of the 1st kind. Using the change of variable $j=n-m$, we have

$$
b_{n, k, j}=(-1)^{k+j-n}(n-1) !(n-j) \frac{s_{j, n-k}}{j ! k !} .
$$

Noting that $s_{j, n-k}=0$ if $j<n-k$ and if $k=0$ or $k=n$, we can write the sum on $j$ from 1 to $n-1$ and the sum on $k$ from 0 to $n$. This leads to the following expression:

$$
\begin{aligned}
& \mathrm{G}_{n}^{(2)}(\mathbf{x})=\left(\frac{\pi}{2}\right)^{n}\left(\frac{\log ^{n}\left(1+x_{1}^{2}\right)}{\left(1+|\mathbf{x}|^{2}\right)^{n+1}}+\frac{(-1)^{n}}{\left(1+x_{1}^{2}\right)^{n}} \sum_{k=1}^{n-1}(-1)^{k} \log ^{k}\left(1+x_{1}^{2}\right) \sum_{m=1}^{k} a_{n, k, m} \frac{\left(1+x_{1}^{2}\right)^{m}}{\left(1+|\mathbf{x}|^{2}\right)^{m+1}}\right) \\
& =\left(\frac{\pi}{2}\right)^{n}\left(\frac{\log ^{n}\left(1+x_{1}^{2}\right)}{\left(1+|\mathbf{x}|^{2}\right)^{n+1}}+(n-1) ! \sum_{k=0}^{n} \sum_{j=1}^{n-1} \frac{s_{j, n-k}}{j ! k !} \frac{(-1)^{j}(n-j)}{\left(1+|\mathbf{x}|^{2}\right)^{n+1-j}\left(1+x_{1}^{2}\right)^{j}} \log ^{k}\left(1+x_{1}^{2}\right)\right) .
\end{aligned}
$$

The structure of the perturbative expansion is similar to the one studied in [30]. In the next section, we will sum the expansion following the same method.

\section{Resummation}

In this section we perform the resummation of the perturbative expansion to obtain an explicit expression for the 2-point function. Let us use the formulas

$$
\begin{aligned}
& (-1)^{j} s_{j, n-k}=\left.\frac{1}{(n-k) !} \frac{\mathrm{d}^{n-k}}{\mathrm{~d} u^{n-k}} \frac{\Gamma(j-u)}{\Gamma(-u)}\right|_{u=0}, \\
& \log ^{k}\left(1+x_{1}^{2}\right)=\left.\frac{\mathrm{d}^{k}}{\mathrm{~d} u^{k}}\left(1+x_{1}^{2}\right)^{u}\right|_{u=0},
\end{aligned}
$$

\footnotetext{
${ }^{2}$ We computed the expansion up to order 9 in the coupling using Mathematica.
} 
to rewrite the second term of the RHS of $(22)$ as

$$
\begin{aligned}
& \left.\left(\frac{\pi}{2}\right)^{n} \sum_{j=1}^{n-1} \frac{n-j}{j ! n} \frac{1}{\left(1+|\mathbf{x}|^{2}\right)^{n+1-j}\left(1+x_{1}^{2}\right)^{j}} \sum_{k=0}^{n}\left(\begin{array}{c}
n \\
k
\end{array}\right)\left(\frac{\mathrm{d}^{n-k}}{\mathrm{~d} u^{n-k}} \frac{\Gamma(j-u)}{\Gamma(-u)}\right)\left(\frac{\mathrm{d}^{k}}{\mathrm{~d} u^{k}}\left(1+x_{1}^{2}\right)^{u}\right)\right|_{u=0} \\
& =\left.\left(\frac{\pi}{2}\right)^{n} \sum_{j=1}^{n-1} \frac{n-j}{j ! n} \frac{1}{\left(1+|\mathbf{x}|^{2}\right)^{n+1-j}\left(1+x_{1}^{2}\right)^{j}} \frac{\mathrm{d}^{n}}{\mathrm{~d} u^{n}}\left(\frac{\Gamma(j-u)}{\Gamma(-u)}\left(1+x_{1}^{2}\right)^{u}\right)\right|_{u=0} .
\end{aligned}
$$

Then using

$$
\begin{aligned}
\frac{\mathrm{d}^{n}}{\mathrm{~d} u^{n}}\left(\frac{\Gamma(j-u)}{\Gamma(-u)}\left(1+x_{1}^{2}\right)^{u}\right) & =\left.\frac{\mathrm{d}^{n}}{\mathrm{~d} u^{n}}\left((-1)^{j}\left(1+x_{1}^{2}\right)^{j} \frac{\mathrm{d}^{j}}{\mathrm{~d}\left(x_{1}^{2}\right)^{j}}\left(1+x_{1}^{2}\right)^{u}\right)\right|_{u=0} \\
& =(-1)^{j}\left(1+x_{1}^{2}\right)^{j} \frac{\mathrm{d}^{j}}{\mathrm{~d}\left(x_{1}^{2}\right)^{j}} \log ^{n}\left(1+x_{1}^{2}\right)
\end{aligned}
$$

and realising that the first term of the rhs of $(22)$ corresponds to $j=0$, we have

$$
\begin{aligned}
\mathrm{G}_{n}^{(2)}(\mathbf{x}) & =\left(\frac{\pi}{2}\right)^{n}\left(\frac{\log ^{n}\left(1+x_{1}^{2}\right)}{\left(1+|\mathbf{x}|^{2}\right)^{n+1}}+\sum_{j=1}^{n-1} \frac{n-j}{j ! n} \frac{(-1)^{j}}{\left(1+|\mathbf{x}|^{2}\right)^{n+1-j}} \frac{\mathrm{d}^{j}}{\mathrm{~d}\left(x_{1}^{2}\right)^{j}} \log ^{n}\left(1+x_{1}^{2}\right)\right) \\
& \left.=\left(\frac{\pi}{2}\right)^{n} \sum_{j=0}^{n-1} \frac{n-j}{j ! n} \frac{(-1)^{j}}{\left(1+|\mathbf{x}|^{2}\right)^{n+1-j}} \frac{\mathrm{d}^{j}}{\mathrm{~d}\left(x_{1}^{2}\right)^{j}} \log ^{n}\left(1+x_{1}^{2}\right)\right) .
\end{aligned}
$$

We then write

$$
\frac{1}{\left(1+|\mathbf{x}|^{2}\right)^{n+1-j}}=\frac{(-1)^{n-j}}{(n-j) !} \frac{\mathrm{d}^{n-j}}{\mathrm{~d}\left(x_{1}^{2}\right)^{n-j}} \frac{1}{\left(1+|\mathbf{x}|^{2}\right)},
$$

to get

$$
\begin{aligned}
\mathrm{G}^{(2)}(\mathbf{x}) & =\frac{1}{1+|\mathbf{x}|^{2}}+\sum_{n=1}^{\infty}\left(\frac{\pi}{2}\right)^{n} \frac{(-1)^{n} \lambda^{n}}{n !} \sum_{j=0}^{n-1}\left(\begin{array}{c}
n-1 \\
j
\end{array}\right) \frac{\mathrm{d}^{n-j}}{\mathrm{~d}\left(x_{1}^{2}\right)^{n-j}} \frac{1}{\left(1+|\mathbf{x}|^{2}\right)} \frac{\mathrm{d}^{j}}{\mathrm{~d}\left(x_{1}^{2}\right)^{j}} \log ^{n}\left(1+x_{1}^{2}\right) \\
& =\frac{1}{1+|\mathbf{x}|^{2}}-\sum_{n=1}^{\infty}\left(\frac{\pi}{2}\right)^{n} \frac{\lambda^{n}}{n !} \frac{\mathrm{d}^{n-1}}{\mathrm{~d}\left(x_{1}^{2}\right)^{n-1}} \frac{\left(-\log \left(1+x_{1}^{2}\right)\right)^{n}}{\left(1+|\mathbf{x}|^{2}\right)^{2}}
\end{aligned}
$$

To sum this series, we use the Lagrange-Bürmann inversion formula [32], [33]. This formula states that for $\phi(\omega)$ analytic at $\omega=0$, such that $\phi(0) \neq 0$ and $f(\omega)=\frac{\omega}{\phi(\omega)}$, the inverse function $g(z)$ of $f(\omega)$, such that $z=f(g(z))$, is analytic at $z=0$ and given by

$$
g(z)=\left.\sum_{n=1}^{\infty} \frac{z^{n}}{n !} \frac{\mathrm{d}^{n-1}}{\mathrm{~d} \omega^{n-1}} \phi(\omega)^{n}\right|_{\omega=0} .
$$

Moreover, for any analytic function $H(z)$ such that $H(0)=0$,

$$
H(g(z))=\left.\sum_{n=1}^{\infty} \frac{z^{n}}{n !} \frac{\mathrm{d}^{n-1}}{\mathrm{~d} \omega^{n-1}}\left(H^{\prime}(\omega) \phi(\omega)^{n}\right)\right|_{\omega=0} .
$$

Hence, for $z=\frac{\pi}{2} \lambda, \phi(\omega)=-\log \left(1+\omega+x_{1}^{2}\right)$ and $H(\omega)=\frac{1}{1+\omega+|\mathbf{x}|^{2}}-\frac{1}{1+|\mathbf{x}|^{2}}$, equation (30) gives

$$
g\left(x_{1}, z\right)=\sum_{n=1}^{\infty} \frac{z^{n}}{n !} \frac{\mathrm{d}^{n-1}}{\mathrm{~d}\left(x_{1}^{2}\right)^{n-1}}\left(-\log \left(1+x_{1}^{2}\right)\right)^{n},
$$


such that

$$
z=-\frac{g\left(x_{1}, z\right)}{\log \left(1+g\left(x_{1}, z\right)+x_{1}^{2}\right)},
$$

which is solved by

$$
g\left(x_{1}, z\right)=z W\left(\frac{1}{z} e^{\frac{1+x_{1}^{2}}{z}}\right)-1-x_{1}^{2}
$$

where $W(z)$ is the Lambert function defined by $z=W\left(z e^{z}\right)$. Then, using equation (31), we can write

$$
\mathrm{G}^{(2)}(\mathbf{x})=\frac{1}{1+|\mathbf{x}|^{2}}-\sum_{n=1}^{\infty} \frac{z^{n}}{n !} \frac{\mathrm{d}^{n-1}}{\mathrm{~d}\left(x_{1}^{2}\right)^{n-1}} \frac{\left(-\log \left(1+x_{1}^{2}\right)\right)^{n}}{\left(1+|\mathbf{x}|^{2}\right)^{2}}=\frac{1}{1+|\mathbf{x}|^{2}+g\left(x_{1}, z\right)} .
$$

This result can be integrated:

$$
\int \mathrm{d} \mathbf{q}_{\hat{1}}\left(G\left(\mathbf{q}_{\hat{c}} x_{1}\right)-\frac{1}{1+\left|\mathbf{q}_{\hat{1}}\right|^{2}}\right)=-\frac{\pi}{4} \log \left(1+x_{1}^{2}+g\left(x_{1}, z\right)\right) .
$$

Using (9) for $c=1$, we recover (33).

We have thus proved that $(35)$ is a solution of the Schwinger-Dyson equation

$$
\mathrm{G}^{(2)}(\mathbf{x})=\left(1+|\mathbf{x}|^{2}+2 \lambda \int \mathrm{d} \mathbf{q}_{\hat{1}}\left(G\left(\mathbf{q}_{\hat{c}} x_{1}\right)-\frac{1}{1+\left|\mathbf{q}_{\hat{1}}\right|^{2}}\right)\right)^{-1} .
$$

In the limit $\lambda \rightarrow 0$, using $W(x)=\log x-\log \log x+o(1)$ we get

$$
\lim _{\lambda \rightarrow 0} \frac{\pi \lambda}{2} W\left(\frac{2}{\pi \lambda} e^{\frac{2\left(1+x_{1}^{2}\right)}{\pi \lambda}}\right)=1+x_{1}^{2},
$$

so that

$$
\lim _{\lambda \rightarrow 0} G^{(2)}(\mathbf{x})=\frac{1}{1+|\mathbf{x}|^{2}},
$$

and we recover the free propagator, as expected.

We established our solution for $\lambda>0$ with $x_{1}, x_{2}, x_{3} \geq 0$ but it can be analytically extended. The Lambert function has many branches behaving differently on the complex plane [34], the branch assignment of our solution depends on $\lambda$. We give a short comment on the holomorphic extension of our solution in $z=\frac{\pi}{2} \lambda$ for a fixed $\mathbf{x}$, which is heavily based on [30 where all the details are discussed.

One first needs to study the map $z \rightarrow z W\left(\frac{1}{z} e^{\frac{1+x_{1}^{2}}{z}}\right)$ to get proposition 15 of $30 \mid$. Taking into account a rescaling of $\frac{2}{\pi}$ to translate their results in term of our $\lambda$, this map is holomorphic on $\mathbb{C} \backslash\left\{-\frac{2}{\pi}\left(1+x_{1}^{2}\right) \frac{\sin \alpha}{\alpha} \mid \frac{\sin \alpha}{\alpha} e^{\alpha \cot \alpha} \geq \frac{\pi e}{2\left(1+x_{1}^{2}\right)},-\pi<\alpha<\pi\right\}$. Varying $x_{1}$, the common holomorphic domain $\Omega$ is at the right of the curve $\mathcal{C}=\left\{-e^{1-\alpha \cot \alpha+i \alpha} \mid-\pi<\alpha<\pi\right\}$ and is not affected by the rescaling. In particular, it contains the disk $|\lambda|<1$ and the map has a convergent radius $\geq 1$ in $\lambda$ for all $x_{1} \geq 0$. In our case, we can have poles if $z W\left(\frac{1}{z} e^{\frac{1+x_{1}^{2}}{z}}\right)=-x_{2}^{2}-x_{3}^{2} \mp i \epsilon=-y \mp i \epsilon$ with $y>0$. This equation can be solved with $\epsilon \rightarrow 0$ by $\lambda_{x_{1}}^{ \pm}(y)$, a critical line in the $z$-plane, parametrised by $y\left(\lambda_{a}^{ \pm}(\varphi)\right.$ in the notation of [30], Lemma 18) and with a specific branch of the Lambert function. For this branch we would get a pole, however the actual branch assignment in our solution for $\lambda=\lambda_{x_{1}}^{ \pm}(y)$ is a different branch and the critical line does not cause any singularity. The two-point function is then holomorphic in the domain $\Omega$ of the complex plane depicted in figure 1 of [30]. 


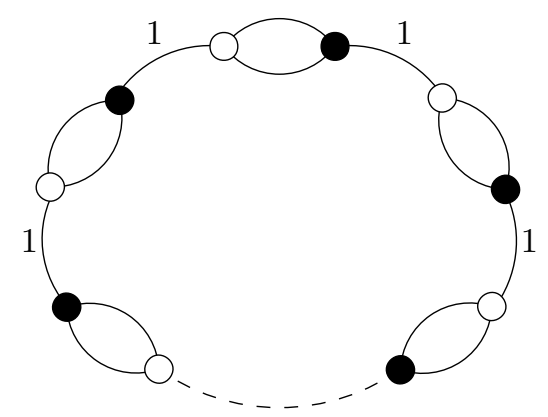

Figure 3: General form of the connected components of boundary graphs in the model with 1 quartic melonic interaction.

\section{$5 \quad$ Higher-point functions}

The boundary graphs of the model with 1 quartic melonic interaction have connected components of the form of figure 3. The $2 k$-point function SDE with connected boundary graph was derived in the section 6 of [25], taking the large $N$ limit established in [31] and explained in section 2, we get

$$
\begin{aligned}
G^{(2 k)}(\mathbf{X}) & =2 \lambda G^{(2)}\left(x_{1}^{1}, x_{2}^{2}, x_{3}^{2}\right) \\
& \times \sum_{\rho=2}^{k} G^{(2 k-2 \rho+2)}\left(\mathbf{x}^{\rho}, \ldots, \mathbf{x}^{k}\right) \frac{G^{(2 \rho-2)}\left(\mathbf{x}^{1}, \ldots, \mathbf{x}^{\rho-1}\right)-G^{(2 \rho-2)}\left(x_{1}^{\rho}, x_{2}^{1}, x_{3}^{1}, \ldots, \mathbf{x}^{\rho-1}\right)}{\left(x_{1}^{1}\right)^{2}-\left(x_{1}^{\rho}\right)^{2}} .
\end{aligned}
$$

From the solution (35) of the 2-point function SDE, we can recursively obtain any higher-point function with a connected boundary graph.

The case of disconnected boundary graph is more involved [26] and no general expression of the SDE in the large $N$ limit have yet been obtained. The simplest equation is the SDE for the 4-point function with disconnected boundary graph, which for only 1 quartic interaction and in the large $N$ limit reduces to

$$
\mathrm{G}_{\mathrm{m} \mid \mathrm{m}}^{(4)}(\mathbf{x}, \mathbf{y})=-2 \lambda\left(\mathrm{G}^{(2)}(\mathbf{x})\right)^{2} \int \mathrm{d} q_{2} \mathrm{~d} q_{3} \mathrm{G}_{\mathrm{m} \mid \mathrm{m}}^{(4)}\left(x_{1}, q_{2}, q_{3}, \mathbf{y}\right)
$$

Analysing the perturbative expansion of $\mathrm{G}_{\mathrm{m} \mid \mathrm{m}}^{(4)}$, we see that there is no contribution at order $\lambda^{0}$, since the Feynman graph which can contribute (made with two free propagator) is disconnected. Moreover in the appendix of [31], we determined that the first contribution to the perturbative expansion is at order $\lambda^{2}$ and corresponds to graphs built with 2 different pillow interactions, of the form of the Feynman graph of figure 1 b). In the present case of the model with only 1 quartic melonic interaction, no such graph exists. Then, by plugging an expansion of the form of (10) for the 2- and 4-point functions in (41), we can recursively establish that all order of the perturbative expansion of $\mathrm{G}_{\mathrm{m} \mid \mathrm{m}}^{(4)}$ are null. Hence, at leading order in the large $N$ limit, $\mathrm{G}_{\mathrm{m} \mid \mathrm{m}}^{(4)}$ is completely suppressed.

\section{Concluding remarks}

In this paper we have solved the 2-point function of a tensor field theory with 1 quartic melonic interaction, with building block the Lambert-W function, using a perturbative expansion and 
a Lagrange-Bürmann resummation. From this result, all higher-point functions with connected boundary graph can be obtained recursively. Moreover, we have shown by a perturbative argument that the 4-point function with a disconnected boundary graph is null at leading order in the large $N$ limit.

A first perspective for this work is the study of higher-point functions with disconnected boundary graph. As mention in section 2, the form of the scalings $\alpha(\mathcal{B})$ is a conjecture made in [31] and based on the study of SDE with connected boundary graph. Now one has to prove this conjecture using the SDE for disconnected boundary graph that have been determined in [26]. As in the connected boundary case, the large $N$ limit of these SDE are expected to involve only lower point function and in particular the 2-point function found in this paper. The fact that the 4-point function with a disconnected boundary graph is null may indicate that at least some of the higher-point functions will also be suppressed at leading order in $N$.

Another perspective which appears interesting to us is the study the model with 3 quartic melonic interactions. The perturbative expansion is more involved but other techniques such as the blobbed topological expansion for such tensor model [35] may prove useful.

\section{Acknowledgement}

The author would like to thank Raimar Wulkenhaar for his guidance throughout this project, Adrian Tanasa for his advice and comments on the manuscript, and Alexander Hock for helpful discussions.

\section{A Recurrence relations}

In this section, we will use the recursive equation 11 to determine recurrence relations on the numbers $a_{n, k, m}$. We first perform the integration

$$
\begin{aligned}
& \int \mathrm{d} \mathbf{q}_{\hat{1}} \mathrm{G}_{p}^{(2)}\left(\mathbf{q}_{\hat{1}} x_{1}\right)=-\frac{\pi}{4} \log \left(1+x_{1}^{2}\right) \quad \text { if } p=0, \\
& =\left(\frac{\pi}{2}\right)^{p+1}\left(\frac{\log ^{p}\left(1+x_{1}^{2}\right)}{2 p\left(1+x_{1}^{2}\right)^{p}}+\frac{(-1)^{p}}{2\left(1+x_{1}^{2}\right)^{p}} \sum_{r=1}^{p-1}(-1)^{r} \log ^{r}\left(1+x_{1}^{2}\right) \sum_{m=1}^{r} \frac{a_{p, r, m}}{m}\right) \quad \text { if } p>0,
\end{aligned}
$$

where for $p=1$ the sum on $r$ does not appear. Plugging back the ansatz $(19)$ in the recurrence relation 111 with $c=1$ gives

$$
\begin{aligned}
& \mathrm{G}_{n}^{(2)}(\mathbf{x})=-\frac{2}{|\mathbf{x}|^{2}+1}\left\{-\frac{\pi}{4} \log \left(1+x_{1}^{2}\right) \mathrm{G}_{n-1}^{(2)}(\mathbf{x})+\sum_{p=1}^{n-1}\left(\frac{\pi}{2}\right)^{p+1} \frac{\log ^{p}\left(1+x_{1}^{2}\right)}{2 p\left(1+x_{1}^{2}\right)^{p}} \mathrm{G}_{n-p-1}^{(2)}(\mathbf{x})\right. \\
& \left.+\sum_{p=2}^{n-1}\left(\frac{\pi}{2}\right)^{p+1}\left(\frac{(-1)^{p}}{2\left(1+x_{1}^{2}\right)^{p}} \sum_{r=1}^{p-1}(-1)^{r} \log ^{r}\left(1+x_{1}^{2}\right) \sum_{m=1}^{r} \frac{a_{p, r, m}}{m}\right) \mathrm{G}_{n-p-1}^{(2)}(\mathbf{x})\right\} .
\end{aligned}
$$

The first term of (44) gives

$$
\frac{\pi \log \left(1+x_{1}^{2}\right)}{2\left(|\mathbf{x}|^{2}+1\right)}\left(\frac{\pi}{2}\right)^{n-1}\left(\frac{\log ^{n-1}\left(1+x_{1}^{2}\right)}{\left(1+|\mathbf{x}|^{2}\right)^{n}}+\frac{(-1)^{n-1}}{\left(1+x_{1}^{2}\right)^{n-1}} \sum_{k=1}^{n-2}(-1)^{k} \log ^{k}\left(1+x_{1}^{2}\right) \sum_{m=1}^{k} a_{n-1, k, m} \frac{\left(1+x_{1}^{2}\right)^{m}}{\left(1+|\mathbf{x}|^{2}\right)^{m+1}}\right)
$$




$$
\begin{aligned}
& =\left(\frac{\pi}{2}\right)^{n}\left(\frac{\log ^{n}\left(1+x_{1}^{2}\right)}{\left(1+|\mathbf{x}|^{2}\right)^{n+1}}+\frac{(-1)^{n-1}}{\left(1+x_{1}^{2}\right)^{n-1}} \sum_{k=1}^{n-2}(-1)^{k} \log ^{k+1}\left(1+x_{1}^{2}\right) \sum_{m=1}^{k} a_{n-1, k, m} \frac{\left(1+x_{1}^{2}\right)^{m}}{\left(1+|\mathbf{x}|^{2}\right)^{m+2}}\right) \\
& =\left(\frac{\pi}{2}\right)^{n}\left(\frac{\log ^{n}\left(1+x_{1}^{2}\right)}{\left(1+|\mathbf{x}|^{2}\right)^{n+1}}+\frac{(-1)^{n}}{\left(1+x_{1}^{2}\right)^{n}} \sum_{k=2}^{n-1}(-1)^{k} \log ^{k}\left(1+x_{1}^{2}\right) \sum_{m=2}^{k} a_{n-1, k-1, m-1} \frac{\left(1+x_{1}^{2}\right)^{m}}{\left(1+|\mathbf{x}|^{2}\right)^{m+1}}\right)
\end{aligned}
$$

where we sent $k \rightarrow k+1$ and $m \rightarrow m+1$ to get to the last line. The second term of (44) gives

$$
\begin{aligned}
& -\frac{2}{|\mathbf{x}|^{2}+1}\left(\sum_{p=1}^{n-1}\left(\frac{\pi}{2}\right)^{p+1} \frac{\log ^{p}\left(1+x_{1}^{2}\right)}{2 p\left(1+x_{1}^{2}\right)^{p}}\left(\frac{\pi}{2}\right)^{n-p-1} \frac{\log ^{n-p-1}\left(1+x_{1}^{2}\right)}{\left(1+|\mathbf{x}|^{2}\right)^{n-p}}\right. \\
& \left.+\sum_{p=1}^{n-3}\left(\frac{\pi}{2}\right)^{p+1} \frac{\log ^{p}\left(1+x_{1}^{2}\right)}{2 p\left(1+x_{1}^{2}\right)^{p}}\left(\frac{\pi}{2}\right)^{n-p-1} \frac{(-1)^{n-p-1}}{\left(1+x_{1}^{2}\right)^{n-p-1}} \sum_{k=1}^{n-p-2}(-1)^{k} \log ^{k}\left(1+x_{1}^{2}\right) \sum_{m=1}^{k} a_{n-p-1, k, m} \frac{\left(1+x_{1}^{2}\right)^{m}}{\left(1+|\mathbf{x}|^{2}\right)^{m+1}}\right) \\
& =-\left(\frac{\pi}{2}\right)^{n} \frac{\log ^{n-1}\left(1+x_{1}^{2}\right)}{\left(1+x_{1}^{2}\right)^{n}} \sum_{p=1}^{n-1} \frac{1}{p} \frac{\left(1+x_{1}^{2}\right)^{n-p}}{\left(1+|\mathbf{x}|^{2}\right)^{n-p+1}} \\
& +\left(\frac{\pi}{2}\right)^{n} \frac{(-1)^{n}}{\left(1+x_{1}^{2}\right)^{n}} \sum_{p=1}^{n-3} \sum_{k=1}^{n-p-2}(-1)^{k-p} \log ^{p+k}\left(1+x_{1}^{2}\right) \sum_{m=1}^{k} \frac{a_{n-p-1, k, m}}{p} \frac{\left(1+x_{1}^{2}\right)^{m+1}}{\left(1+|\mathbf{x}|^{2}\right)^{m+2}} .
\end{aligned}
$$

Setting $r=p+k$ in the line of the previous equation, let us rewrite the double sum as

$$
\sum_{k=1}^{n-3} \sum_{r=k+1}^{n-2} \frac{(-1)^{r}}{r-k} \log ^{r}\left(1+x_{1}^{2}\right) a_{n-r+k-1, k, m}=\sum_{r=2}^{n-2} \sum_{k=1}^{r-1} \frac{(-1)^{r}}{r-k} \log ^{r}\left(1+x_{1}^{2}\right) a_{n-r+k-1, k, m} .
$$

Then we send $m \rightarrow m+1$ and rewrite double sum to get

$$
\sum_{k=1}^{r-1} \sum_{m=2}^{k+1} \frac{a_{n-r+k-1, k, m-1}}{r-k} \frac{\left(1+x_{1}^{2}\right)^{m}}{\left(1+|\mathbf{x}|^{2}\right)^{m+1}}=\sum_{m=2}^{r} \sum_{k=m-1}^{r-1} \frac{a_{n-r+k-1, k, m-1}}{r-k} \frac{\left(1+x_{1}^{2}\right)^{m}}{\left(1+|\mathbf{x}|^{2}\right)^{m+1}} .
$$

Hence, sending $p \rightarrow n-p$ and collecting the results we get

$$
\begin{aligned}
& -\left(\frac{\pi}{2}\right)^{n} \frac{\log ^{n-1}\left(1+x_{1}^{2}\right)}{\left(1+x_{1}^{2}\right)^{n}} \sum_{p=1}^{n-1} \frac{1}{n-p} \frac{\left(1+x_{1}^{2}\right)^{p}}{\left(1+|\mathbf{x}|^{2}\right)^{p+1}} \\
& +\left(\frac{\pi}{2}\right)^{n} \frac{(-1)^{n}}{\left(1+x_{1}^{2}\right)^{n}} \sum_{r=2}^{n-2}(-1)^{r} \log ^{r}\left(1+x_{1}^{2}\right) \sum_{m=2}^{r} \sum_{k=m-1}^{r-1} \frac{a_{n-1+k-r, k, m-1}}{r-k} \frac{\left(1+x_{1}^{2}\right)^{m}}{\left(1+|\mathbf{x}|^{2}\right)^{m+1}} .
\end{aligned}
$$

The third term of (44) gives

$$
\begin{aligned}
& -\frac{2}{|\mathbf{x}|^{2}+1}\left\{\sum_{p=2}^{n-1}\left(\frac{\pi}{2}\right)^{p+1}\left(\frac{(-1)^{p}}{2\left(1+x_{1}^{2}\right)^{p}} \sum_{r=1}^{p-1}(-1)^{r} \log ^{r}\left(1+x_{1}^{2}\right) \sum_{m=1}^{r} \frac{a_{p, r, m}}{m}\right)\left(\frac{\pi}{2}\right)^{n-p-1} \frac{\log ^{n-p-1}\left(1+x_{1}^{2}\right)}{\left(1+|\mathbf{x}|^{2}\right)^{n-p}}\right. \\
& +\sum_{p=2}^{n-3}\left(\frac{\pi}{2}\right)^{p+1}\left(\frac{(-1)^{p}}{2\left(1+x_{1}^{2}\right)^{p}} \sum_{r=1}^{p-1}(-1)^{r} \log ^{r}\left(1+x_{1}^{2}\right) \sum_{m=1}^{r} \frac{a_{p, r, m}}{m}\right) \\
& \left.\left.\left(\frac{\pi}{2}\right)^{n-p-1} \frac{(-1)^{n-p-1}}{\left(1+x_{1}^{2}\right)^{n-p-1}} \sum_{k=1}^{n-p-2}(-1)^{k} \log ^{k}\left(1+x_{1}^{2}\right) \sum_{l=1}^{k} a_{n-p-1, k, l} \frac{\left(1+x_{1}^{2}\right)^{l}}{\left(1+|\mathbf{x}|^{2}\right)^{l+1}}\right)\right\}
\end{aligned}
$$


The first term of equation 50 gives

$$
\begin{aligned}
& -\left(\frac{\pi}{2}\right)^{n} \sum_{p=2}^{n-1} \sum_{r=1}^{p-1}(-1)^{p+r} \frac{\log ^{n-p+r-1}\left(1+x_{1}^{2}\right)}{\left(1+x_{1}^{2}\right)^{n}} \sum_{m=1}^{r} \frac{a_{p, r, m}}{m} \frac{\left(1+x_{1}^{2}\right)^{n-p}}{\left(1+|\mathbf{x}|^{2}\right)^{n-p+1}} \\
& =-\left(\frac{\pi}{2}\right)^{n} \sum_{r=1}^{n-2} \sum_{k=1}^{n-1-r}(-1)^{k} \frac{\log ^{n-k-1}\left(1+x_{1}^{2}\right)}{\left(1+x_{1}^{2}\right)^{n}} \sum_{m=1}^{r} \frac{a_{r+k, r, m}}{m} \frac{\left(1+x_{1}^{2}\right)^{n-r-k}}{\left(1+|\mathbf{x}|^{2}\right)^{n-r-k+1}},
\end{aligned}
$$

by setting $k=p-r$. Then by setting $l=n-1-k$ and rewriting the sums we get

$$
\begin{aligned}
& \left(\frac{\pi}{2}\right)^{n} \frac{(-1)^{n}}{\left(1+x_{1}^{2}\right)^{n}} \sum_{r=1}^{n-2} \sum_{l=r}^{n-2}(-1)^{l} \log ^{l}\left(1+x_{1}^{2}\right) \sum_{m=1}^{r} \frac{a_{n-1+r-l, r, m}}{m} \frac{\left(1+x_{1}^{2}\right)^{l-r+1}}{\left(1+|\mathbf{x}|^{2}\right)^{l-r+2}} \\
& =\left(\frac{\pi}{2}\right)^{n} \frac{(-1)^{n}}{\left(1+x_{1}^{2}\right)^{n}} \sum_{l=1}^{n-2}(-1)^{l} \log ^{l}\left(1+x_{1}^{2}\right) \sum_{r=1}^{l} \sum_{m=1}^{r} \frac{a_{n-1+r-l, r, m}}{m} \frac{\left(1+x_{1}^{2}\right)^{l-r+1}}{\left(1+|\mathbf{x}|^{2}\right)^{l-r+2}}
\end{aligned}
$$

Then we set $k=l-r+1$ and obtain

$$
\left(\frac{\pi}{2}\right)^{n} \frac{(-1)^{n}}{\left(1+x_{1}^{2}\right)^{n}} \sum_{l=1}^{n-2}(-1)^{l} \log ^{l}\left(1+x_{1}^{2}\right) \sum_{k=1}^{l} \sum_{m=1}^{l-k+1} \frac{a_{n-k, l-k+1, m}}{m} \frac{\left(1+x_{1}^{2}\right)^{k}}{\left(1+|\mathbf{x}|^{2}\right)^{k+1}} .
$$

The second term of (50) gives, by rewriting the sums,

$$
\begin{aligned}
& \left(\frac{\pi}{2}\right)^{n} \frac{(-1)^{n}}{\left(1+x_{1}^{2}\right)^{n}} \sum_{p=2}^{n-3} \sum_{k=1}^{n-p-2} \sum_{r=1}^{p-1}(-1)^{k+r} \log ^{k+r}\left(1+x_{1}^{2}\right) \sum_{l=1}^{k} \sum_{m=1}^{r} \frac{a_{p, r, m}}{m} a_{n-p-1, k, l} \frac{\left(1+x_{1}^{2}\right)^{l+1}}{\left(1+|\mathbf{x}|^{2}\right)^{l+2}} \\
& =\left(\frac{\pi}{2}\right)^{n} \frac{(-1)^{n}}{\left(1+x_{1}^{2}\right)^{n}} \sum_{r=1}^{n-4} \sum_{k=1}^{n-3-r}(-1)^{k+r} \log ^{r+k}\left(1+x_{1}^{2}\right) \sum_{l=1}^{k} \sum_{m=1}^{r} \sum_{p=r+1}^{n-2-k} \frac{a_{p, r, m}}{m} a_{n-p-1, k, l} \frac{\left(1+x_{1}^{2}\right)^{l+1}}{\left(1+|\mathbf{x}|^{2}\right)^{l+2}}
\end{aligned}
$$

First by setting $q=k+r$ and by several rewriting of the sums we get

$$
\begin{aligned}
& \left(\frac{\pi}{2}\right)^{n} \frac{(-1)^{n}}{\left(1+x_{1}^{2}\right)^{n}} \sum_{k=1}^{n-4} \sum_{q=k+1}^{n-3}(-1)^{q} \log ^{q}\left(1+x_{1}^{2}\right) \sum_{l=1}^{k} \sum_{m=1}^{q-k} \sum_{p=q-k+1}^{n-2-k} \frac{a_{p, q-k, m}}{m} a_{n-p-1, k, l} \frac{\left(1+x_{1}^{2}\right)^{l+1}}{\left(1+|\mathbf{x}|^{2}\right)^{l+2}} \\
& =\left(\frac{\pi}{2}\right)^{n} \frac{(-1)^{n}}{\left(1+x_{1}^{2}\right)^{n}} \sum_{q=2}^{n-3}(-1)^{q} \log ^{q}\left(1+x_{1}^{2}\right) \sum_{k=1}^{q-1} \sum_{l=1}^{k} \sum_{m=1}^{q-k} \sum_{p=q-k+1}^{n-2-k} \frac{a_{p, q-k, m}}{m} a_{n-p-1, k, l} \frac{\left(1+x_{1}^{2}\right)^{l+1}}{\left(1+|\mathbf{x}|^{2}\right)^{l+2}} \\
& =\left(\frac{\pi}{2}\right)^{n} \frac{(-1)^{n}}{\left(1+x_{1}^{2}\right)^{n}} \sum_{q=2}^{n-3}(-1)^{q} \log ^{q}\left(1+x_{1}^{2}\right) \sum_{l=1}^{q-1} \sum_{k=l}^{q-1} \sum_{m=1}^{q-k} \sum_{p=q-k+1}^{n-2-k} \frac{a_{p, q-k, m}}{m} a_{n-p-1, k, l} \frac{\left(1+x_{1}^{2}\right)^{l+1}}{\left(1+|\mathbf{x}|^{2}\right)^{l+2}} \\
& =\left(\frac{\pi}{2}\right)^{n} \frac{(-1)^{n}}{\left(1+x_{1}^{2}\right)^{n}} \sum_{q=2}^{n-3}(-1)^{q} \log ^{q}\left(1+x_{1}^{2}\right) \sum_{l=2}^{q} \sum_{k=l-1}^{q-1} \sum_{m=1}^{q-k} \sum_{p=q-k+1}^{n-2-k} \frac{a_{p, q-k, m}}{m} a_{n-p-1, k, l-1} \frac{\left(1+x_{1}^{2}\right)^{l}}{\left(1+|\mathbf{x}|^{2}\right)^{l+1}},
\end{aligned}
$$

where we send $l \rightarrow l+1$ in the last line.

Now collecting all the results we obtain recurrence relations on $a_{n, k, m}$ :

$$
a_{n, 1,1}=a_{n-1,1,1}
$$




$$
\begin{aligned}
& a_{n, n-1,1}=\frac{1}{n-1}, \\
& a_{n, n-1, m}=\frac{1}{n-m}+a_{n-1, n-2, m-1}, \quad \text { for } m \in \llbracket 2, n-1 \rrbracket, \\
& a_{n, n-2, m}=a_{n-1, n-3, m-1}+\sum_{r=m-1}^{n-3} \frac{a_{r+1, r, m-1}}{n-2-r}+\sum_{l=1}^{n-1-m} \frac{a_{n-m, n-1-m, l}}{l},
\end{aligned}
$$

for $m \in \llbracket 2, n-2 \rrbracket$,

$$
\begin{aligned}
& a_{n, k, 1}=\sum_{l=1}^{k} \frac{a_{n-1, k, l}}{l}, \quad \text { for } k \in \llbracket 1, n-3 \rrbracket, \\
& a_{n, k, m}=a_{n-1, k-1, m-1}+\sum_{r=m-1}^{k-1} \frac{a_{n-1+r-k, r, m-1}}{k-r}+\sum_{l=1}^{k-m+1} \frac{a_{n-m, k-m+1, l}}{l} \\
& +\sum_{r=m-1}^{k-1} \sum_{l=1}^{k-r} \sum_{p=k-r+1}^{n-2-r} \frac{a_{p, k-r, l} a_{n-p-1, r, m-1}}{l}, \quad \text { for } k \in \llbracket 2, n-3 \rrbracket \text { and } m \in \llbracket 2, k \rrbracket .
\end{aligned}
$$

Rewriting these equations gives explicit relations on Stirling numbers of the first kind, harmonic numbers and binomial coefficients. Indeed, from equation 60 we recover

$$
\frac{1}{(n-1) !}\left[\begin{array}{l}
n-1 \\
n-k
\end{array}\right]=\sum_{l=1}^{k} \frac{1}{(n-l) !}\left[\begin{array}{l}
n-1-l \\
n-1-k
\end{array}\right], \quad \text { for } k \in \llbracket 1, n-3 \rrbracket,
$$

which correspond to the equation (6.21) in [36]. Setting $l=n-2-r, k=n-m-1$ and sending $n-3 \rightarrow n$, equation 59 gives

$$
H_{k}=\frac{k+1}{2 n+3-k} \sum_{l=1}^{k} \frac{n+1-k+l}{l(k+1-l)}, \quad \text { for } k \in \llbracket 1, n \rrbracket .
$$

Sending $r \rightarrow k-l$ and in the last term $l \rightarrow r$ of equation (61), we get

$$
\begin{aligned}
& ((n-1) m-k(m-1)) \frac{(n-2) !}{k !(n-m) !}\left[\begin{array}{c}
n-m \\
n-k
\end{array}\right] \\
& =\sum_{l=1}^{k-m+1} \frac{1}{(n-m-l) !}\left[\begin{array}{c}
n-m-l \\
n-k-1
\end{array}\right]\left(\frac{(n-1-m) !}{(k-m+1) !}+\frac{m-1}{l} \frac{(n-l-2) !}{(k-l) !}\right) \\
& +\sum_{l=1}^{k-m+1} \frac{m-1}{l !(k-l) !} \sum_{p=l+1}^{n-2-k+l} \frac{(p-1) !(n-2-p) !}{(n-m-p) !}\left[\begin{array}{c}
n-m-p \\
n-k-1-p+l
\end{array}\right] \sum_{r=1}^{l} \frac{1}{(p-r) !}\left[\begin{array}{c}
p-r \\
p-l
\end{array}\right],
\end{aligned}
$$

for $k \in \llbracket 2, n-3 \rrbracket$ and $m \in \llbracket 2, k \rrbracket$.

\section{References}

[1] R. Gurau, "Colored Group Field Theory," Commun. Math. Phys., vol. 304, pp. 69-93, 2011. DOI: $10.1007 / \mathrm{s} 00220-011-1226-9$, arXiv: 0907.2582 [hep-th].

[2] S. Carrozza and A. Tanasa, "O(N) Random Tensor Models," Lett. Math. Phys., vol. 106, no. 11, pp. 1531-1559, 2016. DOI: $10.1007 /$ s11005-016-0879-x. arXiv: 1512.06718 [math-ph]. 
[3] D. Benedetti, S. Carrozza, R. Gurau, and M. Kolanowski, "The $1 / N$ expansion of the symmetric traceless and the antisymmetric tensor models in rank three," Commun. Math. Phys., vol. 371, no. 1, pp. 55-97, 2019. DOI: 10.1007/s00220-019-03551-z. arXiv: 1712.00249 [hep-th].

[4] R. Gurau, Random Tensors. Oxford University Press, 2017.

[5] E. Witten, "An SYK-Like Model Without Disorder," J. Phys. A, vol. 52, no. 47, p. 474002 , 2019. DOI: 10.1088/1751-8121/ab3752, arXiv: 1610.09758 [hep-th].

[6] I. R. Klebanov and G. Tarnopolsky, "Uncolored random tensors, melon diagrams, and the Sachdev-Ye-Kitaev models," Phys. Rev., vol. D95, no. 4, p. 046 004, 2017. DoI: 10. 1103/PhysRevD.95.046004, arXiv: 1611.08915 [hep-th].

[7] S. Sachdev and J. Ye, "Gapless spin fluid ground state in a random, quantum Heisenberg magnet," Phys. Rev. Lett., vol. 70, p. 3339, 1993, arXiv:cond-mat/9212030.

[8] A. Kitaev, "A simple model of quantum holography," KITP, 2015, [Online]. Available: http://online.kitp.ucsb.edu/online/entangled15/kitaev.

[9] J. Maldacena and D. Stanford, "Remarks on the Sachdev-Ye-Kitaev model," Phys. Rev., vol. D94, no. 10, p. 106 002, 2016. DOI: 10.1103/PhysRevD.94.106002, arXiv: 1604 . 07818 [hep-th].

[10] D. J. Gross and V. Rosenhaus, "All point correlation functions in SYK," JHEP, vol. 12, p. 148, 2017. DOI: 10.1007/JHEP12(2017)148. arXiv: 1710.08113 [hep-th].

[11] R. Gurau, "The complete 1/N expansion of a SYK-like tensor model," Nucl. Phys., vol. B916, pp. 386-401, 2017. DOI: 10.1016/j.nuclphysb.2017.01.015. arXiv: 1611.04032 [hep-th].

[12] V. Bonzom, L. Lionni, and A. Tanasa, "Diagrammatics of a colored SYK model and of an SYK-like tensor model, leading and next-to-leading orders," J. Math. Phys., vol. 58, no. 5, p. 052301,2017 . DOI: 10.1063/1.4983562, arXiv: 1702.06944 [hep-th].

[13] I. R. Klebanov, F. Popov, and G. Tarnopolsky, "TASI Lectures on Large $N$ Tensor Models," PoS, vol. TASI2017, p. 004, 2018. DOI: 10.22323/1.305.0004. arXiv: 1808 . 09434 [hep-th].

[14] N. Delporte and V. Rivasseau, "The Tensor Track V: Holographic Tensors," in 17TH HELLENIC SCHOOL AND WORKSHOPS ON ELEMENTARY PARTICLE PHYSICS AND GRAVITY, Apr. 2018. arXiv: 1804.11101 [hep-th].

[15] D. Benedetti and R. Gurau, "2PI effective action for the SYK model and tensor field theories," JHEP, vol. 05, p. 156, 2018. DOI: 10.1007/JHEP05(2018)156. arXiv: 1802 . 05500 [hep-th].

[16] J. Ben Geloun and V. Rivasseau, "A Renormalizable 4-Dimensional Tensor Field Theory," Commun. Math. Phys., vol. 318, pp. 69-109, 2013. DOI: 10.1007/s00220-012-1549-1. arXiv: 1111.4997 [hep-th].

[17] S. Carrozza, "Flowing in Group Field Theory Space: a Review," SIGMA, vol. 12, p. 070, 2016. DOI: 10.3842/SIGMA.2016.070, arXiv: 1603.01902 [gr-qc].

[18] S. Carrozza, "Tensorial methods and renormalization in Group Field Theories," PhD thesis, Orsay, LPT, 2014. DOI: $10.1007 / 978-3-319-05867-2$, arXiv: 1310.3736 [hep-th]. 
[19] J. Ben Geloun and V. Rivasseau, "A Renormalizable SYK-type Tensor Field Theory," Annales Henri Poincare, vol. 19, no. 11, pp. 3357-3395, 2018. DOI: $10.1007 /$ s00023018-0712-4. arXiv: 1711.05967 [hep-th].

[20] A. Eichhorn, J. Lumma, T. Koslowski, and A. D. Pereira, "Towards background independent quantum gravity with tensor models," Classical and Quantum Gravity, vol. 36, no. 15, p. 155 007, Jul. 2019. DOI: 10.1088/1361-6382/ab2545. arXiv: 1811.00814 [gr-qc].

[21] A. Eichhorn, T. Koslowski, and A. D. Pereira, "Status of background-independent coarsegraining in tensor models for quantum gravity," Universe, vol. 5, no. 2, p. 53, 2019. DOI: 10.3390/universe5020053, arXiv: 1811.12909 [gr-qc].

[22] T. Krajewski and R. Toriumi, "Exact Renormalisation Group Equations and Loop Equations for Tensor Models," SIGMA, vol. 12, p. 068, 2016. DOI: 10.3842/SIGMA.2016.068. arXiv: 1603.00172 [gr-qc].

[23] D. O. Samary, "Closed equations of the two-point functions for tensorial group field theory," Class. Quant. Grav., vol. 31, p. 185 005, 2014. DOI: 10.1088/0264-9381/31/ 18/185005. arXiv: 1401.2096 [hep-th].

[24] C. I. Pérez-Sánchez, "The full Ward-Takahashi Identity for colored tensor models," Commun. Math. Phys., vol. 358, no. 2, pp. 589-632, 2018. DoI: 10.1007/s00220-018-3103-2. arXiv: 1608.08134 [math-ph].

[25] R. Pascalie, C. I. Pérez-Sánchez, and R. Wulkenhaar, "Correlation functions of U $(N)$ tensor models and their Schwinger-Dyson equations," 2017. arXiv: 1706.07358 [math-ph].

[26] C. I. Pérez-Sánchez, "Graph calculus and the disconnected-boundary Schwinger-Dyson equations in tensor field theory," 2018. arXiv: 1812.00623 [math-ph].

[27] D. Ousmane Samary, C. I. Pérez-Sánchez, F. Vignes-Tourneret, and R. Wulkenhaar, "Correlation functions of a just renormalizable tensorial group field theory: the melonic approximation," Class. Quant. Grav., vol. 32, no. 17, p. 175012 , 2015. DOI: 10.1088/ 0264-9381/32/17/175012, arXiv: 1411.7213 [hep-th].

[28] M. Disertori, R. Gurau, J. Magnen, and V. Rivasseau, "Vanishing of Beta Function of Non Commutative Phi**4(4) Theory to all orders," Phys. Lett., vol. B649, pp. 95-102, 2007. DOI: 10.1016/j . physletb.2007.04.007, arXiv: hep-th/0612251 [hep-th].

[29] H. Grosse and R. Wulkenhaar, "Self-Dual Noncommutative $\phi^{4}$-Theory in Four Dimensions is a Non-Perturbatively Solvable and Non-Trivial Quantum Field Theory," Commun. Math. Phys., vol. 329, pp. 1069-1130, 2014. DOI: 10.1007/s00220-014-1906-3. arXiv: 1205.0465 [math-ph].

[30] E. Panzer and R. Wulkenhaar, "Lambert-W Solves the Noncommutative $\Phi^{4}$-Model," Commun. Math. Phys., vol. 374, no. 3, pp. 1935-1961, 2019. Dor: 10.1007 /s00220019-03592-4. arXiv: 1807.02945 [math-ph].

[31] R. Pascalie, C. I. Pérez-Sánchez, A. Tanasa, and R. Wulkenhaar, "On the large N limit of Schwinger-Dyson equations of a rank-3 tensor field theory," Journal of Mathematical Physics, vol. 60, no. 7, p. 073 502, Jul. 2019. Dor: 10.1063/1.5080306. arXiv: 1810.09867 [math-ph].

[32] J. L. Lagrange, "Nouvelle méthode pour résoudre des équations littérales par le moyen de séries," Mém. Acad. Roy. des Sci. et Belles-Lettres de Berlin, vol. 24, 1770.

[33] H. Bürmann, "Essai de calcul fonctionnaire aux constantes ad-libitum," Mem.Inst. Nat. Sci Arts. Sci. Math. Phys., vol. 2, pp. 316-347, 1799. 
[34] R. M. Corless, G. H. Gonnet, D. E. G. Hare, D. J. Jeffrey, and D. E. Knuth, "On the lambertw function," Advances in Computational Mathematics, vol. 5, no. 1, pp. 329-359, Dec. 1996. DOI: $10.1007 / \mathrm{BF} 02124750$.

[35] V. Bonzom and S. Dartois, "Blobbed topological recursion for the quartic melonic tensor model," J. Phys., vol. A51, no. 32, p. 325 201, 2018. DOI: 10.1088/1751-8121/aac8e7. arXiv: 1612.04624 [hep-th].

[36] R. L. Graham, D. E. Knuth, and O. Patashnik, Concrete mathematics: A foundation for computer science, 2nd. Boston, MA, USA: Addison-Wesley Longman Publishing Co., Inc., 1994. 